\title{
Resiliência à mudança climática em Comunidades de Fundo de Pasto na região semiárida do Estado da Bahia, Brasil
}

\author{
Larisa Ho Bech Gaivizzo ${ }^{1}$ \\ Gabriela Litre ${ }^{1}$ \\ Julia Lopes Ferreira ${ }^{1}$ \\ Romero Gomes Pereira da Silva ${ }^{1}$ \\ Daniela Nogueira Soares ${ }^{1}$ \\ Rafael Moraes Reis ${ }^{1}$ \\ Ana Claudia Almeida ${ }^{1}$ \\ Nelson Eduardo Bernal Davalos ${ }^{1}$ \\ Priscylla Dayse Almeida Gonçalves Mendes ${ }^{1}$ \\ Diego Pereira Lindoso ${ }^{1}$ \\ Adriane Michels Brito ${ }^{1}$ \\ Saulo Rodrigues-Filho ${ }^{1}$ \\ Carlos Hiroo Saito ${ }^{1}$
}

\begin{abstract}
Resumo
A pesquisa realizada entre 2017 e 2018 buscou produzir conhecimentos que subsidiem ações voltadas à resiliência e à adaptação de sistemas socioecológicos na região semiárida do estado da Bahia - Semiárido Baiano, com foco nas Comunidades de Fundo de Pasto (CFP) localizadas no município de Uauá. Utilizaram-se dados secundários e primários (entrevistas semiestruturadas com tomadores de decisão e lideranças comunitárias). Para a sistematização dos dados primários no marco de capacidades adaptativas para mudanças climáticas foram selecionadas cinco dimensões de análise: territorial, sociocultural, ambiental, econômica e político-institucional. As CFP ocupam e usam o bioma Caatinga há cerca de 200 anos de forma comunal, com base em sistemas agrossilvopastoris. Os resultados indicaram o caráter resiliente das comunidades para lidar com os estresses climáticos nas dimensões territorial e sociocultural, a partir de processos de auto-organização em associações e na organização em redes, por meio das quais fluem conhecimentos e são tomadas decisões coletivas. Os resultados também indicaram os riscos climáticos aos quais as CFP estão expostas nas dimensões ambiental, econômica e políticoinstitucional, com potencial para limitar capacidades adaptativas.
\end{abstract}

Palavras-chave: Sistemas socioecológicos. Capacidade adaptativa. Comunidades agrossilvopastoris. Decisões coletivas.

\footnotetext{
${ }^{1}$ UnB - University of Brasília, Brasília, Distrito Federal, Brazil. larisabech@gmail.com Artigo recebido em: 13/12/2018. Aceito para publicação em: 19/07/2019.
} 


\begin{abstract}
The objective of this study was to determine how to support actions to increase the resilience and adaptation of socio-ecological systems in the semiarid region of Bahia State, focusing on the Fundo de Pasto Communities (FPCs) of the Uauá municipality. Primary (semistructured interviews with decision-makers and community leaders) and secondary data sources were used. Five dimensions of analysis were selected to organize the primary data within a framework of adaptive capacity (AC) for climate change: territorial, socio-cultural, environmental, economic and politico-institutional. For approximately 200 years, FPCs have been communally occupying and using the Caatinga biome based on agrosilvopastoral systems. The results showed the communities' resilience in dealing with climate stresses in both the territorial and socio-cultural dimensions. This includes processes of self-organization in associations and in networks, which facilitate the flow of knowledge and collective decision-making processes. The results also identified the environmental, economic and politico-institutional dimensions of the climate risks to FPCs, which could potentially limit their AC.

Keywords: socio-ecological systems, adaptive capacity, agrosilvopastoral communities, collective decisions.
\end{abstract}

\title{
Introdução
}

Os primeiros registros de períodos de seca na região do Semiárido Brasileiro remontam ao século XVI (SILVA et al., 2017). O último deles, considerado um dos mais intensos e extensos desde a década de 1960, ocorreu de 2012 a 2017 (MARENGO et al., 2016, 2018; SILVA et al., 2017). As secas recorrentes tendem a se agravar no contexto das mudanças climáticas. Os modelos climáticos do Painel Intergovernamental sobre Mudanças Climáticas (IPCC) $(2007,2014)$ projetam para a região um aquecimento entre $1,5^{\circ} \mathrm{C}$, em um cenário otimista, e $5^{\circ} \mathrm{C}$, no cenário mais pessimista, até o ano de 2100. Indicam, ainda, redução da precipitação anual, intensificação das secas e diminuição da disponibilidade de água para o consumo humano, animal e vegetal (PBMC, 2013). Estes cenários colocam em risco os sistemas sociais e ambientais locais, cujos modos de vida dependem diretamente da Caatinga, bioma predominante no Semiárido (MARENGO, 2006; ROCHA, 2009). Dessa maneira, reconhece-se a exacerbação da vulnerabilidade desses sistemas às mudanças climáticas. 
A vulnerabilidade é função de três atributos - exposição, sensibilidade e capacidade adaptativa, sendo que: i) a exposição e a sensibilidade indicam a suscetibilidade dos sistemas aos impactos e riscos climáticos; e, ii) a capacidade adaptativa reflete as condições ou as possibilidades em lidar com esses impactos e riscos, tanto absorvendo-os (resiliência), como pelo ajuste às mudanças que ocasionam (adaptação) (ADGER; BROWN, 2009; FOLKE, 2016; LINDOSO; RODRIGUES-FILHO, 2016). Tendo em vista essas definições, este estudo analisa elementos comuns da capacidade adaptativa que caracterizam dinâmicas coevolutivas em sistemas socioecológicos - a partir de longas trajetórias de interação entre as comunidades humanas e os ecossistemas, em regiões semiáridas.

\section{As Comunidades de Fundo de Pasto}

As Comunidades de Fundo de Pasto (CFP) são de difícil definição devido à sua heterogeneidade (FERRARO, 2008; SANTOS, 2011). Em termos gerais, podem ser identificadas como comunidades que adotam sistemas de pastoreio comunitário extensivo de animais de pequeno porte (especialmente caprinos). Na região do Semiárido Brasileiro localizada no estado da Bahia - o Semiárido Baiano (SAB), há cerca de 200 anos (FERRARO, 2008), as CFP se territorializaram, usando a terra de forma comunal (FERRARO; BURSZTYN; DRUMMOND, 2017; GARCEZ, 1987).

As áreas comunais das CFP são originárias tanto de sesmarias e fazendas abandonadas, datadas dos séculos XVIII e XIX, como provenientes de terras devolutas, ocupadas por famílias associadas à figura dos vaqueiros, índios e negros escravizados, e de uma parcela da população livre empobrecida (REIS, 2015). Nessas terras os grupos produziram e acumularam ao longo do tempo valores de comunidades camponesas (relações de terra-propriedade-família) e tradicionais (apossamentos 
coletivos da terra, relação dos sujeitos com o território), e fazendo surgir o uso comunal da terra (MARQUES, 2016; TORRES, 2011).

Até o momento, o governo do estado da Bahia reconheceu formalmente 373 comunidades (GEOGRAFAR, 2018). Contudo, com base em levantamentos de pesquisa documental junto a organizações sociais baianas, estima-se que existam mais de 500 CFP ocupando uma área com cerca de 1,2 milhões de hectares que englobam 20 mil famílias rurais, distribuídas em 52 municípios do estado (FERRARO, 2008; REIS, 2015).

Essas áreas comunais das CFP desenvolvem a caprinocultura com o direito de uso comum da pastagem nativa da Caatinga e o extrativismo vegetal, principalmente de umbu (Spondias tuberosa), mas também de maracujá (Passiflora cincinnata), licuri (Syagrus coronata), angico (Anadenanthera macrocarpa) e murici (Byrsonima crassifolia), entre outras espécies (CARVALHO, 2014; SABOURIN; CARON; SILVA, 1999).

\section{Material e Métodos}

Para a análise da coevolução de capacidade adaptativa em CFP foram identificadas variáveis qualitativas em cinco dimensões: i) trajetórias de territorialização (dimensão territorial); ii) capacidade de organização e fluxos de conhecimentos (dimensão sociocultural), iii) manejo de recursos comuns (dimensão ambiental); iv) diversificação da renda de produção (dimensão econômica); v) acesso a políticas públicas e flexibilidade das instituições estatais (dimensão político-institucional) (BALBO et al., 2016; BENNET et al., 2016; FOLKE, 2016; LANCELOTTI et al., 2016; SACHS, 2002; WILSON, 2014).

A pesquisa com base em dados secundários e primários foi realizada entre 2017 e 2018, com uma abordagem multimetodológica que combina técnicas quantitativas (como o levantamento de dados em bases 
governamentais e a espacialização de indicadores) e qualitativas (como entrevistas semiestruturadas) (TEDDLIE; TASHAKKORI, 2009). O desenvolvimento do estudo foi aprovado pelo Comitê de Ética em Pesquisa em Ciências Humanas e Sociais da Universidade de Brasília (UnB) processo número 98071318.0.0000.5540.

Foram realizadas reuniões técnicas, seminários, oficinas de trabalho com representantes da Articulação no Semiárido Brasileiro (ASA), do Comitê de Bacia Hidrográfica do Rio São Francisco, da Empresa Brasileira de Pesquisa Agropecuária (Embrapa Semiárido), da Universidade Federal do Vale do São Francisco (Univasf), do Ministério do Meio Ambiente (MMA) e do Ministério do Desenvolvimento Social e Combate à Fome (MDS).

Nessas atividades, realizaram-se também entrevistas com 23 membros das CFP, líderes locais de diferentes comunidades no município de Uauá (BA). O município, que concentra as CFP no SAB, possui aproximadamente 5\% de sua área total inserida na Bacia Hidrográfica do rio São Francisco (IBGE, 2010) (Mapa 1). Entre os líderes locais, quatro são também representantes das comunidades em instituições regionais: Fundo e Feixo de Pasto (Articulação Estadual de Fundo de Pasto); Cooperativa de Agropecuária Familiar de Canudos, Uauá e Curaçá (COOPERCUC); o Instituto Regional da Pequena Agropecuária Apropriada (IRPAA); e a Câmara Consultiva Regional do Submédio rio São Francisco do Comitê de Bacia Hidrográfica do Rio São Francisco. 
Mapa 1 - Espacialização dos locais das entrevistas com os líderes das Comunidades de Fundo de Pasto no município de Uauá no Semiárido Baiano.

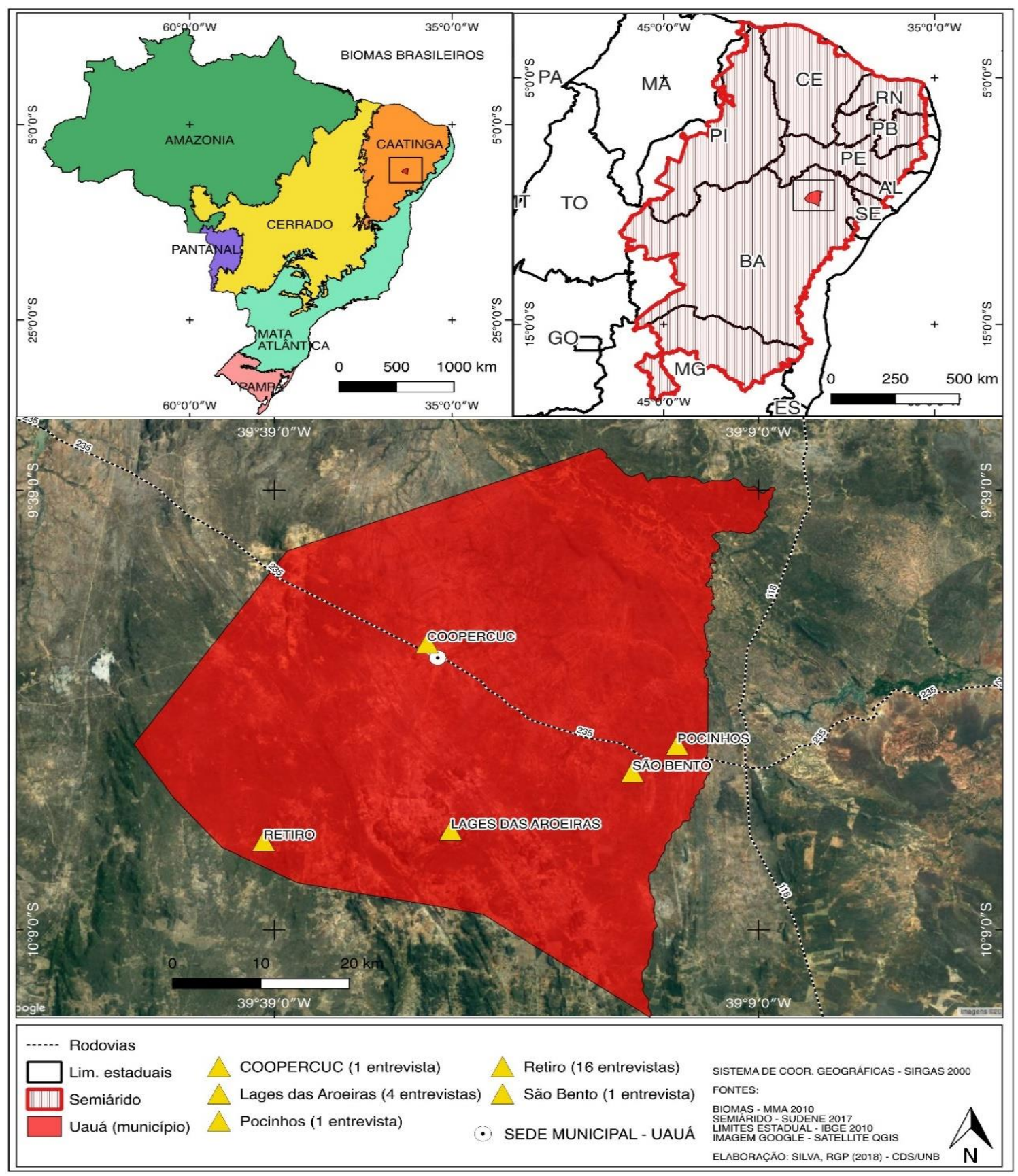

A análise das entrevistas apoiou-se em: i) leituras sucessivas das narrativas dos atores a partir das anotações de campo, a fim de identificar percepções-chaves sobre cada tema; ii) sistematização das informações de campo nas dimensões e variáveis qualitativas de análise; iii) análise das 
percepções-chaves à luz de abordagens conceituais e empíricas da literatura científica acrescidas de dados secundários complementares.

\section{Resultados e Discussão}

\section{Relações entre a Caatinga e CFP: desafios}

O Mapa 2 mostra a distribuição espacial das 373 CFP certificadas na Bahia, aquelas formalmente reconhecidas pelo governo do estado (GEOGRAFAR, 2018). Conforme mencionado, o município de Uauá (BA), é o território que apresenta a maior concentração de comunidades certificadas (53). Na dimensão territorial, a análise da variável trajetórias de territorialização nas escalas espacial e temporal concorda com Ferraro (2008), que diz que a distribuição das CFP coincide geograficamente com a localização dos antigos currais das sesmarias baianas - Casa da Torre e Casa da Ponte. Isso indica a existência de elementos de resistência das CFP no $\mathrm{SAB}$ e coevolução da capacidade adaptativa relacionados à variável capacidade de organização na dimensão sociocultural.

A organização das CFP se deu a partir dos conflitos de terra nas décadas de 1970 e 1980 (MARQUES, 2016). No final da década de 1980, o instrumento de Concessão de Direito Real de Uso é adotado pelo estado da Bahia como estratégia para se construir a segurança fundiária (CARVALHO, 2014). A Concessão é celebrada entre o estado e associações "legalmente" instituídas das CFP. Esses processos culminaram na formação de 599 associações de Fundo de Pasto, na criação das Centrais e da Articulação Estadual de CFP (com caráter de representação política) e da Cooperativa de Agropecuária Familiar de Canudos, Uauá e Curaçá (COOPERCUC, com caráter de inclusão socioprodutiva dos FP) (CARVALHO, 2014; TORRES, 2011). Observa-se que cada comunidade frequentemente possui mais de uma associação. As associações expressam 
as formas de organização na escala local e são os elos entre as famílias das CFP e os movimentos sociais na escala regional.

Mapa 2 - Distribuição das Comunidades de Fundo de Pasto no estado da Bahia.

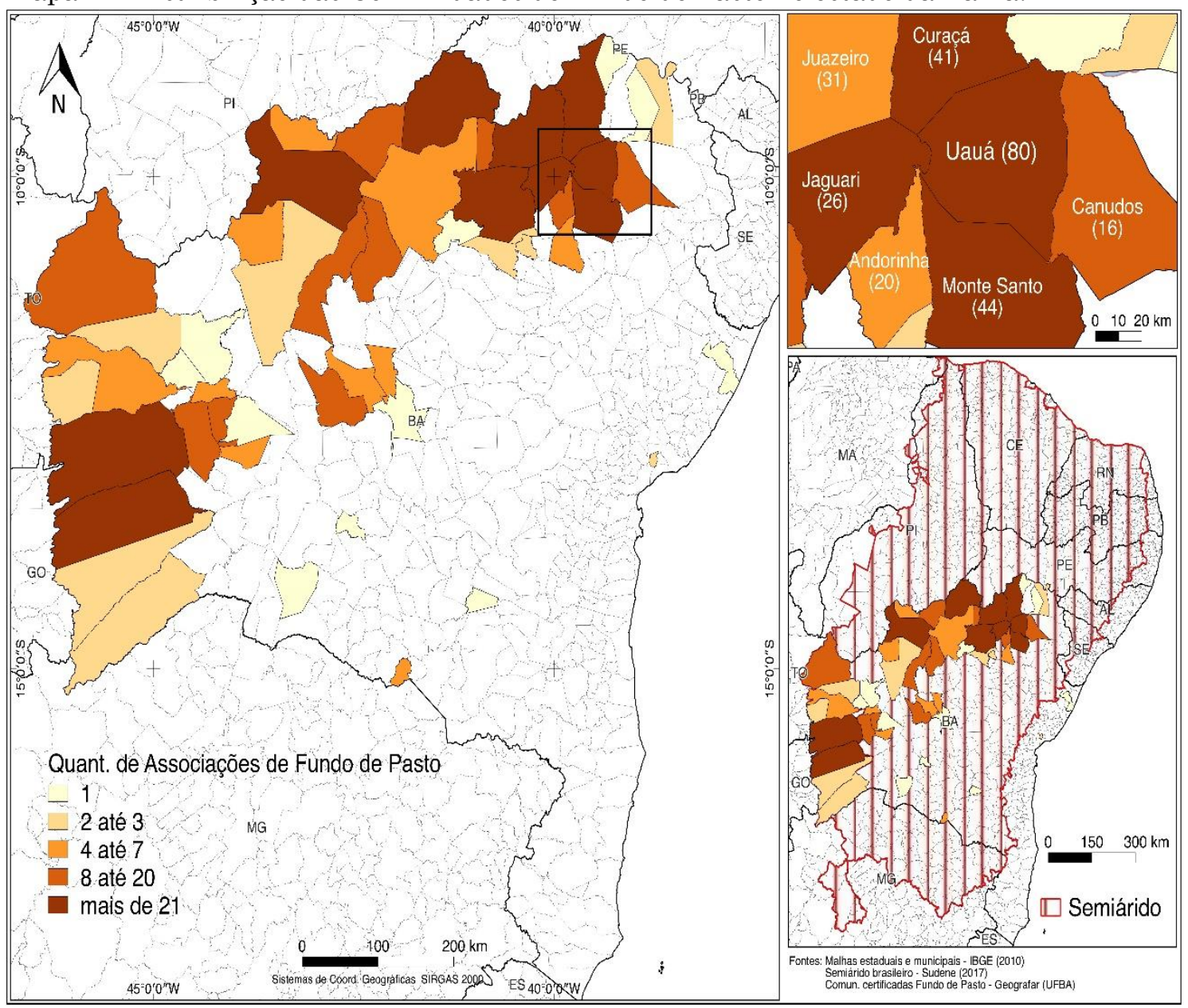

O Mapa 3 faz referência a um levantamento realizado por Carvalho (2014) junto aos líderes de CFP sobre a representatividade das diferentes formas de organização das comunidades. Nota-se que a COOPERCUC, criada em 2004, tem papel relevante para a representação política das comunidades. O IRPAA, protagonista político e sociotécnico junto às CFP, é a organização social responsável pela introdução do cooperativismo no município de Uauá e nos municípios do entorno. A constituição da Cooperativa é apontada pelos entrevistados como um ponto de inflexão 
econômica das famílias de Fundo de Pasto, especialmente dos jovens e das mulheres.

Mapa 3 - Representações simbólicas das organizações sociais junto às Comunidades de

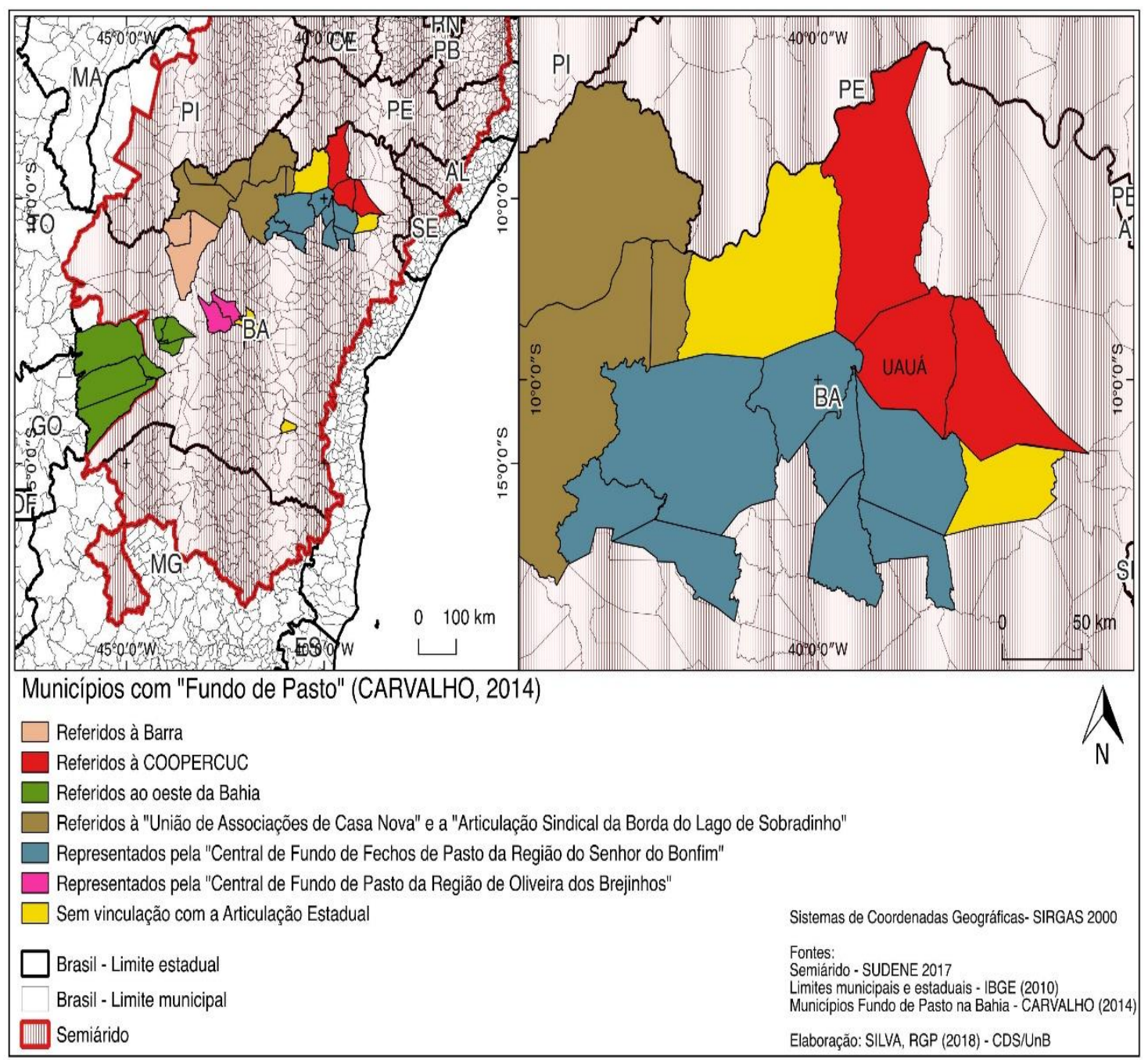

Fundo de Pasto.

A ASA um papel-chave para a conectividade das organizações que representam os CFP no $\mathrm{SAB}$ e, portanto, para o planejamento e as decisões no Semiárido Brasileiro e nacional. Sua criação, na década de 1990, decorre da emergência do paradigma da "Convivência com o Semiárido", em oposição ao paradigma de “Combate às Secas" (LINDOSO et al., 2018), a partir das demandas sociais de organizações locais e regionais (PORTO; FROELICH, 2018). Atualmente, a ASA é integrada por mais de mais de 
1.000 organizações que atuam em rede, gerando e disseminando tecnologias adequadas ao Semiárido. Estima-se que cerca de 144 tecnologias já foram desenvolvidas no âmbito da rede (RODRIGUES, 2016). Muitas dessas tecnologias estão fundamentadas na cultura de estocagem de água (como cisternas de consumo e de produção), na produção de sementes (como os bancos de sementes crioulas) e no plantio de forrageiras como a palma (Opuntia ficus-indica Mill), utilizada para a suplementação da alimentação dos caprinos (LINDOSO et al., 2018; PETTERSEN; SILVEIRA, 2017).

A capacidade de organização das CFP baseada no associativismo (e, mais recentemente, no cooperativismo) e a priorização do trabalho em rede constituem um recurso adaptativo que vem, nos últimos 200 anos (FERRARO, 2008; PORTO; FROELICH, 2018; SANTOS, 2011), coevoluindo no SAB junto à territorialização das CFP.

Nesse amplo tecido de organizações sociais, estrutura-se outro recurso de capacidade adaptativa na dimensão sociocultural, central para o Fundo de Pasto: o fluxo multidirecional de experiências e conhecimentos tradicionais, técnicos e científicos entre as escalas comunitária e regional. Por meio da rede, as CFP aprendem, armazenam e compartilham conhecimentos para lidar com o estresse climático. Elas também tomam decisões coletivas, de caráter policêntrico, mediadas por instituições com capacidade de fornecer respostas flexíveis frente às incertezas, e desenvolvem soluções locais para os problemas (BALBO et al., 2016; IPCC, 2014; MARSHALL et al., 2010).

Um exemplo de solução ancorada nesse fluxo multidirecional de trocas apontada pelos entrevistados são as redes de segurança alimentar e fundiária. A segurança alimentar se manifesta na intensificação da troca de alimentos e sementes crioulas entre as comunidades, por meio de suas associações, para assegurar a subsistência das famílias durante os períodos de seca. A segurança fundiária se expressa no fortalecimento das regras coletivas que inibem a venda das posses privadas para pessoas externas à 
comunidade, movimento que tende a ocorrer nesses períodos, conforme apontado por Ferraro (2008).

Destaca-se que a defesa dos direitos territoriais ainda é um elemento de coesão entre as organizações das CFP, com desafios que perpassam e articulam todas as dimensões deste estudo (CARVALHO, 2014; MARQUES, 2016; SANTOS, 2011).

Tais desafios referem-se tanto à lentidão por parte do poder público na regularização fundiária das áreas comunais, como à natureza do instrumento de regularização, a Concessão de Direito Real de Uso, segundo a qual a propriedade da terra coletiva continua sendo pública. Em uma das comunidades visitadas, Comunidade do Retiro (Mapa 1), que tem 28 mil hectares de área coletiva e está sob a gestão de 600 famílias de CFP, somente cerca de 2 mil hectares estão regularizados. Além disso, o governo estadual determinou que a regularização do restante das terras deveria ser feita até dezembro de 2018, tempo insuficiente diante da proporção de territórios ainda não reconhecidos.

Vale assinalar que, segundo os entrevistados, ao mesmo tempo que empreende esforços para a regularização das áreas comunais, o estado da Bahia também tem autorizado estudos de prospecção de minérios em áreas não regularizadas, o que representa uma das principais fontes de ameaça externa às CFP e de conflitos entre políticas públicas. Reis (2015) cita que diversas empresas já realizaram o mapeamento mineral no município de Monte Santo (BA), fronteiriço a Uauá e detentor da segunda maior concentração de comunidades reconhecidas (Mapa 2) e associações de CFP no estado.

Tanto as variáveis na dimensão territorial como na dimensão sociocultural têm como elo central a interdependência das CFP com a Caatinga (PEREZ-MARIN et al., 2017; ROCHA, 2009). Dada a riqueza de seus recursos genéticos e endêmicos, o manejo de recursos comuns na Caatinga - ou seja, a variável qualitativa da dimensão ambiental - 
desempenha um papel crucial na sustentabilidade das CFP (FERRARO; BURSZTYN; DRUMMOND, 2017). Em outras palavras, economias rurais de pequena escala podem existir em um ambiente sob estresse climático devido a essa biodiversidade. A combinação de conhecimento tradicional e gestão do bioma dá origem a um conjunto de práticas bem articuladas, integradas à cultura das CFP, informadas pelos longos períodos de observação e convivência com esse ambiente (FERNANDEZ-LLAMAZARES et al., 2016; PYHALA et al., 2016).

Isso significa que os usos da terra nas comunidades se fundamentam na "saúde" e na (re)produção da Caatinga, os quais asseguram os seus modos de vida. Vários pesquisadores apontam que, ao longo de sua existência, as CFP vêm mantendo a diversidade da vegetação da Caatinga, visando o uso comum do território e o manejo do potencial nativo da vegetação para a obtenção de renda, alimento, água, medicamentos, energia, sementes e matéria-prima para as mais variadas finalidades, principalmente nos períodos de escassez hídrica (PORTO; FROELICK, 2018; SABOURIN; CARON; SILVA, 1999; SANTOS, 2011). A vegetação da Caatinga tem características que refletem a evolução em condições semiáridas (ROCHA, 2009; SEYFFARTH; RODRIGUES, 2016).

Nesse escopo, um dos relatos de maior relevância dos líderes das CFP relacionados aos impactos da última seca foi a observação do recente ciclo de morte de plantas nativas da Caatinga. Além de uma redução de quase 40\% da população dos umbuzeiros, os líderes mencionam a redução da população das macambiras (Bromelia laciniosa), uma espécie endêmica do bioma. A redução da cobertura vegetal afeta, por exemplo, as espécies de fauna polinizadoras e dispersoras de sementes, fundamentais para a reprodução do bioma (SEYFFARTH; RODRIGUES, 2016). Ademais, a Caatinga carece de políticas adequadas para a sua conservação (ROCHA, 2009).

Existe um consenso sobre isso nas narrativas locais, assim como sobre os efeitos negativos para a alimentação animal (com perda de matrizes) e 
para o extrativismo vegetal. A renda da produção agrossilvopastoril fundamenta-se na criação de caprinos nas pastagens naturais da Caatinga e nos produtos desse bioma, especialmente o umbu, que é a matéria-prima principal da COOPERCUC, comercializado na forma de doces, geleias e cerveja. Diante desse quadro, na área comunal da CFP de 28 mil hectares anteriormente citada, as famílias realizaram o cercamento de parte desse espaço, de modo a restringir o acesso dos caprinos e permitir a regeneração do bioma.

Cabe aqui detalhar como as CFP organizam o uso dos recursos naturais, bem como suas estratégias produtivas e o caráter adaptativo das práticas agroextrativistas no território. O estabelecimento de regras coletivas para o uso de recursos comuns reforçadas nos períodos de seca prevê situações que potencialmente poderiam derivar na "tragédia dos comuns" retratada por Hardin (1968), que analisa a tendência natural das pessoas em maximizar os benefícios próprios em detrimento de benefícios coletivos. A partir de estudos empíricos em comunidades, Ostrom (1990), em contraposição à visão de Hardin, indica que, quando adotados princípios para a gestão compartilhada dos recursos comuns, tais como o estabelecimento de limites claros de uso, a equivalência entre benefícios e custos, decisões coletivas, sanções gradativas, resolução justa de conflitos, autonomia comunitária e governança bottom up, o comportamento das pessoas distancia-se do individualismo. Os dados deste estudo sobre as CFP mostram que elas representam um exemplo concreto em favor de Ostrom, em que a organização coletiva e comunal reforça a cooperação e a governança.

A dimensão ambiental ganha relevância na análise das capacidades adaptativas em contexto de mudanças climáticas. Neste cenário, emergem das pesquisas de campo elementos presentes na literatura sobre possíveis fatores que podem estar afetando a estabilidade de algumas espécies vegetais nativas da Caatinga nas CFP. Entre eles, o aumento na população 
dos rebanhos de caprinos e os períodos sucessivos de seca e sua intensificação (LITRE et al., 2018; MARENGO et al., 2018; SEYFFARTH; RODRIGUES, 2017).

Um levantamento realizado por Lindoso et al. (2018) aponta um aumento de $83 \%$ do número de caprinos na Bacia Hidrográfica do Rio São Francisco (parte do município de Uauá está na Bacia) no período da última seca. Litre et al. (2018) atribui esse fenômeno à preferência dos pecuaristas por esses animais, cujos rebanhos demonstram ser mais resistentes à seca. Ferraro (2008) mostra o sistema sazonal de alimentação dos caprinos nas CFP, no qual os frutos de umbu têm papel relevante nos meses de janeiro a abril, e a macambira, em fevereiro e de agosto a novembro, o que poderia explicar um possível aumento de pressão do rebanho sobre os recursos naturais.

O umbuzeiro possui nas suas raízes órgãos tuberosos (xilopódios), chamados localmente de "cucas", os quais armazenam nutrientes e água. São reservas estratégicas que nutrem a planta mesmo em anos de seca severa. Apesar disso, Seyffarth e Rodrigues (2017) relatam que a morte dos umbuzeiros ainda não tinha sido observada em ciclos de seca anteriores aos de 2010 a 2017. Eles relacionam o fenômeno à intensificação da seca num contexto de mudanças climáticas (MARENGO et al., 2016; 2018; PBMC, 2013; SILVA et al., 2017).

A cadeia de impactos dos processos de alterações climáticas associados a práticas de manejo inadequadas e a suscetibilidade de desertificação no Semiárido se expressam na degradação dos solos e dos recursos hídricos, na degradação da vegetação e da biodiversidade e, em última análise, na redução da qualidade de vida da população dependente da Caatinga (DOURADO, 2017; MARENGO, 2006). Além disso, a projeção de uma significativa redução das chuvas até 2100 pode provocar profundas alterações no funcionamento do ecossistema (PBMC, 2013). 
No marco da dimensão econômica sobre a variável diversificação da renda produtiva, observa-se que a elevada dependência das CFP em relação à base de recursos naturais confere uma baixa elasticidade na composição da renda. Tendo em vista que a extração e o beneficiamento do umbu são realizados principalmente por mulheres, que representam mais de $60 \%$ dos sócios da COOPERCUC, é possível considerar a clivagem de gênero como um atributo de sensibilidade diferencial neste estudo.

Um aspecto destacado pelos entrevistados é que a renda oriunda dos produtos da biota, além da inclusão socioprodutiva das mulheres, fortaleceu o seu protagonismo político. Assim, no caso das mulheres sócias da COOPERCUC, suas estratégias de inclusão no mercado de trabalho e de incremento da renda parecem ser as primeiras a sofrer tais impactos.

Vale dizer que tais impactos não se restringem à sua inclusão socioprodutiva, mas afetam também a participação desses grupos nos processos decisórios de suas comunidades. É interessante perceber que, mais uma vez, os grupos socialmente mais vulneráveis são aqueles mais afetados pela intensificação de fenômenos multiescalares, como as mudanças climáticas (ADGER; BROWN, 2009; NOGUEIRA, 2017).

$\mathrm{Na}$ dimensão político-institucional, os líderes das CFP indicam a redução do acesso às políticas de educação, crédito e extensão rural. E, quando acessadas, a concepção das instituições na esfera pública (elaborada de forma dissociada da complexidade territorial, sociocultural e ambiental do Semiárido) frequentemente se traduz em uma baixa flexibilidade institucional, reforçando processos produtivos que, no médio prazo, degradam o bioma e desestruturam o uso comum das terras pelas CFP (PEREZ-MARIN et al., 2017; REIS, 2015).

O desenho de políticas públicas adequadas aos contextos sociais no SAB e o posterior acesso a elas são essenciais para a regulação das dinâmicas de interdependência entre as CFP e a Caatinga. A sustentação das atividades produtivas ao longo do tempo depende da manutenção do 
fluxo dos serviços ecossistêmicos (de provisão, regulação e suporte) desse bioma (MARENGO, 2006; MEA, 2005).

\section{Capacidade adaptativa das CFP}

O estudo das percepções dos líderes das CFP mostrou evidências empíricas sobre possíveis caminhos (e lições) para adaptação e resiliência de sistemas socioecológicos no $\mathrm{SAB}$ a partir do desenvolvimento de respostas coletivas na escala local. A coevolução de capacidades adaptativas no sistema socioecológico das CFP pode ser observada no reconhecimento do sistema secular de ocupação e uso comunal do território por meio do estabelecimento de regras coletivas para a gestão dos recursos comuns. Mais recentemente, as estratégias de adaptação das CFP evoluíram para a autoorganização em associações e a organização em redes territoriais. Por meio das redes ocorre o fluxo multidirecional de conhecimentos, com processos de tomada de decisões de caráter policêntrico.

Santos (2011) e Marques (2016) salientam o perfil político das CFP, que no transcorrer das lutas pelas terras comunais impulsionaram sua identidade como um grupo culturalmente diferenciado, sob a alcunha de Fundo de Pasto, que possui formas próprias de organização social facilitadas por relações familiares e de compadrio. Essas trajetórias têm gerado instituições sociais adaptadas aos estresses climáticos no $\mathrm{SAB}$, as quais desenvolvem e disseminam aprendizados, inovações e tecnologias para a convivência com a região.

As lições das CFP identificadas principalmente nas dimensões territorial e sociocultural estão amplamente corroboradas na literatura científica (FOLKE, 2016; GÒMEZ-BAGGETHUM et al., 2012; IUCN, 2008, 2009; OSTROM, 1990) e são elementos comuns da capacidade adaptativa que caracterizam dinâmicas coevolutivas em regiões semiáridas (BALBO et al., 2014; MARSHALL et al., 2010; REYES-GARCIA et al., 2017). 
A partir das percepções levantadas, nota-se que a relação com a Caatinga perpassa as diferentes facetas da capacidade adaptativa e as articula frente aos desafios enfrentados pelas comunidades. Essa capacidade tem como fundamento a utilização da diversidade da Caatinga, por sucessivas gerações, para enfrentar as secas (FERNANDEZ-LLAMAZARES et al., 2016; PYHALA et al., 2016).

Entretanto, os resultados também mostram os riscos aos quais as pessoas na escala local estão expostas, considerando sobretudo o aumento da velocidade e a intensidade de fatores de mudanças multiescalares (ADGER; BROWN, 2009; BENNET et al., 2016). Vale lembrar que a seca de 2010 a 2017 foi uma das mais severas dos últimos 60 anos. A Caatinga, apesar de resistente a condições edafoclimáticas semiáridas, já sofre um pronunciado estresse hídrico, com reduzida taxa de sequestro e baixo estoque de carbono, números que poderão ser ainda menores com a intensificação das secas, resultando em profundas alterações em sua estrutura e serviços ecossistêmicos (PBMC, 2013). Essas mudanças podem impactar a habilidade construída pelas comunidades e afetar sua capacidade de resposta aos estresses climáticos (BALBO et al., 2016).

A redução da população dos umbuzeiros é um indicador relevante para a dimensão ambiental. Sistemas sociais dependentes dos recursos da biodiversidade são mais sensíveis aos impactos e riscos climáticos. Conforme essa dependência aumenta, os sistemas sociais tornam-se mais vulneráveis às alterações do clima (ELLIS, 2000; MARSHAL et al., 2010). Nesse cenário, a análise nas dimensões econômica e político-institucional indica que: a baixa diversificação da renda produtiva; as restrições e os retrocessos ao acesso dos recursos sociotécnicos e financeiros de políticas públicas voltadas à produção agrossilvopastoril e à conservação da Caatinga, aliados a uma visão das instituições estatais dissociada da complexidade do Semiárido, limitam a capacidade das CFP de lidar com mudanças multiescalares. 
A convergência de percepções dos líderes das CFP sobre o retrocesso nas políticas federais, consideradas fundamentais para o enfrentamento da última seca, como é o caso de cisternas de produção (Programa Uma Terra e Duas Águas - P1+2) e mercados institucionais (Programa de Aquisição de Alimentos - PAA), é outro indicador relevante na dimensão políticoinstitucional. Para eles, o retrocesso das políticas está relacionado à redução das dotações orçamentárias e a alterações em seus desenhos metodológicos, que desconsideram importantes componentes sociais que contribuíam para a construção de estratégias adaptativas (NOGUEIRA, 2017; PORTO; FROELICK; 2018).

Os líderes reconhecem também que essas políticas voltadas para a produção diferem das políticas de proteção (Programa Bolsa Família) e previdência social (aposentadoria rural), centrais na renda das famílias de CFP. Pode-se dizer, assim, concordando com Marques (2016), que as CFP desenvolveram uma conscientização coletiva estruturando as suas condições de transformação enquanto sujeitos políticos para o enfrentamento da expansão do capital mercantil regional a partir de seu histórico de enfrentamento dos conflitos pela terra.

A análise da resiliência enquanto capacidade dos sistemas humanos e ambientais de absorver impactos está associada à noção de uma qualidade positiva da capacidade adaptativa e pode ser indicada pela extensão, pelo equilíbrio e pela sobreposição das dimensões territorial, sociocultural, ambiental, econômica e político-institucional (LANCELOTTI et al., 2016; WILSON, 2014). Assim, o caráter resiliente das CFP parece estar centrado em sua dimensão sociocultural e territorial (desde que os direitos territoriais dessas comunidades sejam assegurados), sendo que nas dimensões ambiental, econômica e político-institucional podem tanto aumentar a resiliência como acelerar sua desestabilização.

Se por um lado os caminhos de territorialização e os usos da terra são elementos centrais para a adaptação das CFP, por outro vários fatores 
concorrem para o aumento da sua exposição e sensibilidade, e redução da capacidade adaptativa às mudanças climáticas. Neste sentido, o modo como essas comunidades aprenderam a conviver com o Semiárido pode fornecer importantes lições para a resiliência em sistemas adaptativos complexos (BALBO et al., 2016; FOLKE, 2016; LANCELOTTI et al., 2016). Isso justifica a avaliação de que as CFP constituem um locus privilegiado para o estudo da resiliência, vulnerabilidade e adaptação local às mudanças climáticas em áreas semiáridas.

\section{Considerações finais}

As CFP constituem um importante repositório de iniciativas locais para lidar com os estresses climáticos. As iniciativas vêm se fortalecendo ao longo do tempo em sua dimensão territorial e sociocultural, mas estão vulneráveis em sua dimensão ambiental, econômica e político-institucional, frequentemente em função de forças externas à escala local, conformando cenários futuros com baixa resiliência às mudanças climáticas.

A capacidade das instituições estatais e a sustentabilidade das políticas públicas são elementos cruciais. Em paralelo, a sintonia do enraizamento cultural com as práticas das CFP é essencial para a capacidade adaptativa. E, finalmente, na esfera da territorialidade, a capacidade adaptativa dos CFP é uma garantia da permanência de suas populações na atividade rural, frente a um contexto de forte êxodo para a cidades, com todas as suas implicações.

Os serviços ambientais prestados pelas CFP podem e devem ser valorizados de diversas formas, como por meio de pagamento pela sua conservação. Mas é importante alertar que, por serem sistemas sociais dependentes dos recursos da biodiversidade, as CFP são mais sensíveis aos riscos climáticos, os quais aumentam à medida que essa dependência aumenta. Dessa maneira, pode-se dizer que a vulnerabilidade aos estresses 
climáticos é multifacetada, e o presente estudo sobre as CFP reforça esse modo de compreensão do tema.

\section{Agradecimentos}

Os autores agradecem o apoio intelectual e técnico da Subrede de Desenvolvimento Regional da Rede Brasileira de Pesquisa em Mudanças Climáticas e do Projeto INCT-ODISSEIA "Observatório das Dinâmicas Socioambientais", coordenados pelo Centro de Desenvolvimento Sustentável (CDS) da UnB. Assim como o apoio financeiro do CNPq, da FAP-DF e da CAPES.

\section{Referências}

ADGER, W. N.; BROWN, K. Vulnerability and Resilience to Environmental Change: Ecological and Social Perpectives. In: Global Environmental Change. JANSSEN, M.A.; OSTROM, E. (Eds.). Elsevier. 2006. v.16. p.235-316.

BALBO, A. L. et al. Resilience of small-scale societies: a view from drylands. Ecology and Society, v.21, n.2, p. art53, 2016. https://doi.org/10.5751/ES-08327-210253

BENNETT, N. J. et al. Communities and change in the anthropocene: understanding social-ecological vulnerability and planning adaptations to multiple interacting exposures. Regional Environmental Change, v.16, n.4, p.907-926, 2016. https://doi.org/10.1007/s10113-015-0839-5

CARVAlHO, F. P. D. Fundos de Pasto: territorialidade, luta e reconhecimento. Dissertation (Doctoral Degree), Brasília: Universidade Federal da Bahia, 2014.

DOURADO, C. DA S. Áreas de risco de desertificação: cenários atuais e futuros, frenteàs mudanças climáticas. Dissertation (Doctoral Degree), Campinas: Unicamp, 2017.

ELLIS, F. The Determinants of Rural Livelihood Diversification in Developing Countries.

Journal Of Agricultural Economics, v.51, n.2, p.289-302, May. 2000. https://doi.org/10.1111/j.1477-9552.2000.tb01229.x

FERNÁNDEZ-LLAMAZARES, Á. et al. Local perceptions as a guide for the sustainable management of natural resources: Empirical evidence from a small-scale society in Bolivian Amazonia. Ecology and Society, v.21, n.1, 2016. https://doi.org/10.5751/ES-08092-210102 FERRARO, L. A. J. Entre a invenção da tradição e a imaginação da sociedade sustentável: estudo de caso dos fundos de pasto na Bahia. Dissertation (Doctoral Degree), Brasília: Universidade de Brasília, 2008.

FERRARO, L. A.; BURSZTYN, M.; DRUMMOND, J. A. Sustainability of the remaining agricultural commons in the Brazilian northeast: Challenges beyond management. Erde, v.148, n.2-3, p.150-166, 2017.

FOLKE, C. Resilience (Republished). Ecology and Society, v.21, n.4, 2016.

GARCEZ, A. Fundo de Pasto: um projeto de vida sertanejo. Bahia: INTERBA/SEPLANTEC/CAR, Salvador, 1987. 
GEOGRAFAR. Formas de acesso à terra no Estado da Bahia. Banco de Dados, 2018. Available in: <www.geografar.ufba.br > . Acesso em: 10 maio 2018.

HARDIN, G. The Tragedy of the Commons. Science, 162: 1243-1248, 1968. https://doi.org/10.1126/science.162.3859.1243

IBGE - Instituto Brasileiro de Geografia e Estatística. Censo Demográfico 2010. Rio de Janeiro: IBGE, 2010.

INSA - Instituto Nacional do Semiárido. Estabelecimentos agropecuários do Semiárido Brasileiro. Campina Grande (PB): INSA, 2017.

IPCC - Intergovernmental Panel on Climate Change. Climate Change 2014: Synthesis Report. Contribution of Working Groups I, II and III to the Fifth Assessment Report of the Intergovernmental Panel on Climate Change [Core Writing Team, R.K. Pachauri and L.A. Meyer (Eds.)]. IPCC, Geneva, Switzerland, 2014.

.Fourth Assessment Report: Climate Change 2007. Cambrigde, 2007.

IUCN. International Union for the Conservation of Nature. Indigenous and Traditional People and Climate Change. Issues Paper. IUCN: Gland, 2008. 66 p.

LANCELOTTI, C. et al. Resilience of small-scale societies' livelihoods: A framework for studying the transition from food gathering to food production. Ecology and Society, v.21, n.4, 2016. https://doi.org/10.5751/ES-08757-210408

LINDOSO, D.; RODRIGUES FILHO, S. Vulnerabilidade e adaptação: bases teóricas e conceituais da pesquisa. In: Marcel Bursztyn \& Saulo Rodrigues Filho (Eds.). O clima em transe: vulnerabilidade e adaptação da agricultura familiar. Rio de Janeiro: Garamond. p. 19-34, 2016.

LINDOSO, D.; LITRE, G.; GAIVIZZO, L.; RODRIGUES-FILHO, S.; REIS, J. C. Vulnerabilidade climática da produção rural no semiárido: reflexões para o debate sobre desenvolvimento sustentável e agronegócio. In: Sustentabilidade e Agronegócio. ANDREOLI, C.; PHILIPPI JR., A. (Eds.). São Paulo: Editora Manole. Coleção ambiental (em avaliação).

LITRE, G.; LINDOSO, D.; GAIVIZZO, L.; RODRIGUES-FILHO, S. Vulnerabilidade e Adaptação Climática da Pecuária Familiar no Bioma Caatinga: Limites e Potencialidades do Paradigma de Convivência com o Semiárido. Resumo expandido. Third International Conference "Agriculture and Food in a urbanizing society". Porto Alegre, UFRGS, 2018.

LITTLE, P. E. Territórios sociais e povos tradicionais no Brasil: por uma antropologia da territorialidade. Anuário Antropológico, p. 251-290, 2004.

MARENGO, J. A. Mudanças climáticas globais e seus efeitos sobre a biodiversidade: caracterização do clima atual e definição das alterações climáticas para o território brasileiro ao longo do século XXI. Brasília, DF: Ministério do Meio Ambiente, 2006. 159 p. (Biodiversidade, 26).

MARENGO, J. A., TORRES, R. R., ALVES L. M. Drought in Northeast Brazil - past, present, and future. Theor Appl Climatol, p.1-12, 2016.

MARENGO et al. Climatic characteristics of the 2010-2016 drought in the semiarid Northeast Brazil region. Anais da Academia Brasileira de Ciências, v.90, n.2, p.9731985, 2018. https://doi.org/10.1590/0001-3765201720170206

MARQUES, L. de S. As comunidades de fundo de pasto: um intento de construção conceitual. Pegada. A Revista da Geografia do Trabalho, v.17, n.2, 2016. https://doi.org/10.33026/peg.v17i2.4706

MARSHALL, N. A. et al. A Framework for Social Adaptation to Climate Change: Sustaining Tropical Coastal Communitites and Industries. IUCN, 2010.

MEA - Millennium Ecosystem Assessment. Ecosystems and Human Well-Being: Synthesis. Washington, DC: Island Press, 137 p., 2005.

NOGUEIRA, D. Segurança Hídrica, Adaptação e Gênero: o caso das cisternas para captação de água de chuva no semiárido brasileiro. Revista Sustentabilidade em Debate, Dossiê Água e Gênero, v.8, n.3, 2017. https://doi.org/10.18472/SustDeb.v8n3.2017.26544 
OSTROM, E. Governing the Commons: the evolution of institutions for collective action.

Indiana University, University Press, Cambridge, 1990. https://doi.org/10.1017/CBO9780511807763

PBMC - Painel Brasileiro de Mudanças Climáticas. Contribuição do Grupo de Trabalho 1 ao Primeiro Relatório de Avaliação Nacional do Painel Brasileiro de Mudanças Climáticas. Sumário Executivo GT1. Rio de Janeiro: PBMC, 2013. 24 p.

PÉREZ-MARIN, A. M. et al. Agroecological and social transformations for coexistence with semi-aridity in Brazil. Sustainability (Switzerland), v.9, n.6, p.1-17, 2017. https://doi.org/10.3390/su9060990

PETERSEN, P. F.; SILVEIRA, L. M. Agroecology, public policies and labor-driven intensification: Alternative development trajectories in the brazilian semi-arid region. Sustainability (Switzerland), v.9, n.4, 2017. https://doi.org/10.3390/su9040535

PORTO, S. FROELICH, G. Articulação para o Semiárido Brasileiro. Relatório. Rio de Janeiro: ASA, 2018 (documento interno).

PYHÄLÄ, A. et al. Global environmental change: local perceptions, understandings, and explanations. Ecology and Society, v.21, n. 3, p. 25, 2016. https://doi.org/10.5751/ES08482-210325

REIS, F. S. Associativismo em comunidades de fundo de pasto no município de Monte Santo (BA): mobilização social, dinâmica de poder. Thesis (Master Degree), Cachoeira: Universidade Federal do Recôncavo da Bahia. 2015.

REYES-GARCÍA, V. et al. Small-scale societies and environmental transformations: Coevolutionary dynamics. Ecology and Society, v.22, n.1, p.1-6, 2017. https://doi.org/10.5751/ES-09066-220115

ROCHA, W. F. Situação da cobertura vegetal do bioma Caatinga. In: ANGELOTTI, F.; SÁ, I. B.; MENEZES, E. A.; Pellegrino, G. Q. (Orgs.). Mudanças climáticas e desertificação no Semiárido brasileiro. Petrolina: Embrapa Semiárido; Campinas: Embrapa Informática Agropecuária, 2009. cap. 5, p.77-94.

RODRIGUES, R. S. O caminho das águas: tecnologias de convivência com o semiárido e transições sociotécnicas no sertão brasileiro. Thesis (Master Degree). Universidade Federal Rural do Rio de Janeiro, Instituto de Ciências Humanas e Sociais. 180 f. 2016.

SABOURIN, E.; CARON, P.; SILVA, P. C. G. DA. O manejo dos "fundos de pasto" no nordeste baiano: um exemplo de reforma agraria sustentável. Raízes - Revista de Ciências Sociais e Econômicas, v.18, n.2, p.90-102, 1999.

SACHS, I. Caminhos para o desenvolvimento sustentável. Rio de Janeiro: Garamond, 2002.

SANTOS, C. J. S. E. Fundo de pasto - tecitura da resistência, rupturas e permanências no tempo-espaço desse modo de vida camponês. Dissertation (Doctoral Degree), São Paulo: Universidade de São Paulo, 25 fev.2011.

SEYFFARTH, A. S.; RODRIGUES, V.Impactos da seca sobre a biodiversidade da Caatinga. Parc. Estrat., v.22, n.44, p.41-62, 2017.

SILVA, A.R.S. et al. Variações no Índice de Anomalia de Chuva no Semiárido. Journal of Environmental Analysis and Progress, v.2, n.4, p.377-384, 2017. TEDDLIE, C., TASHAKKORI, A. Foundations of Mixed Methods Research: Integrating Quantitative and Qualitative Approaches in the Social and Behavioral Sciences. California (USA): SAGE, 2009.

TORRES, P. R. Terra e territorialidade das áreas de fundo de pasto no semiárido baiano. Thesis (Master Degree), Salvador: Universidade Católica de Salvador. 2011.

WILSON, G. A. Community resilience: path dependency, lock-in effects and transitional ruptures, Journal of Environmental Planning and Management, 57:1, 1-26, 2014. https://doi.org/10.1080/09640568.2012.741519 